\title{
Language Processing Within the Human Medial Temporal Lobe
}

\author{
Patric Meyer, ${ }^{1,2}$ Axel Mecklinger, ${ }^{1}$ Thomas Grunwald, ${ }^{3,4}$ Juergen Fell, ${ }^{4}$ Christian E. Elger, \\ and Angela D. Friederici ${ }^{5}$
}

\begin{abstract}
Although the hippocampal formation is essential for verbal memory, it is not fully understood how it contributes to language comprehension. We recorded event-related potentials (ERPs) directly from two substructures of the medial temporal lobe (MTL), the rhinal cortex and the hippocampus proper, while epilepsy patients listened to sentences that either were correct or contained semantic or syntactic violations. Semantic violations elicited a large negative ERP response peaking at $\sim \mathbf{4 0 0} \mathbf{~ m s}$ in the rhinal cortex. In contrast, syntactically incorrect sentences elicited a negative deflection of $500-800 \mathrm{~ms}$ in the hippocampus proper. The results suggest that functionally distinct aspects of integration in language comprehension are supported by different MTL structures: the rhinal cortex is involved in semantic integration, whereas the hippocampus proper subserves processes of syntactic integration. An analysis of phase synchronization within the gamma band between rhinal and hippocampal recording sites showed that both of the above-mentioned ERP components were preceded by an increase of phase synchronization. In contrast to these short phasic increases of phase synchronization in both violation conditions, correct sentences were associated with a long-lasting synchronization in a late time window, possibly reflecting the integration of semantic and syntactic information as required for normal comprehension. $\odot 2005$ Wiley-Liss, Inc.
\end{abstract}

KEY WORDS: $\quad$ hippocampus; rhinal cortex; syntax; semantics; binding

\section{INTRODUCTION}

Despite the diversity of theories on the medial temporal lobe (MTL) memory system, the essential role of the hippocampal formation in declarative memory performance remains undisputed. Its involvement in particular processes of language comprehension, however, is less well understood. For example, testing of language functions in patient H.M. revealed a mixed pattern of results (MacKay et al., 1998): although his general language abilities appear to be intact (Kensinger et al., 2001), he exhibits a distinct performance deficit, especially appearing in the detection and understanding of ambiguities. It is argued that switching to the nonpreferred syntactical structure or to the nonpreferred second meaning of a word in syntactic and semantic ambiguous sentences is achieved by forming new connections between single items. This process is hypothesized to involve input from binding nodes within the hippocampus

${ }^{1}$ Department of Psychology, Experimental Neuropsychology Unit, Saarland University, Saarbrücken, Germany; ${ }^{2}$ Department of Neurology II, Otto von Guericke University, Magdeburg, Germany; ${ }^{3}$ Swiss Epilepsy Center, Zurich, Switzerland; ${ }^{4}$ Department of Epileptology, University of Bonn, Bonn, Germany; ${ }^{5}$ Max Planck Institute for Human Cognitive and Brain Sciences, Leipzig, Germany

*Correspondence to: Patric Meyer, Department of Psychology, Saarland University, Experimental Neuropsychology Unit, P.O. Box 1511 50, Saarbrücken D-66041, Germany. E-mail: patricmeyer1@aol.com

Accepted for publication 14 December 2004

DOI 10.1002/hipo.20070

Published online 15 February 2005 in Wiley InterScience (www.interscience. wiley.com).
(MacKay et al., 1998) However, until now it is unclear what the specific processing function of the hippocampus during language comprehension might be, and whether its involvement is necessary in semantic as well as syntactic integration.

Another way to investigate the involvement of the hippocampal formation in language comprehension is to use intracranial ERP recordings. Such recordings from the temporal lobes of epilepsy patients showed a large negative potential with a mean latency of $\sim 400$ $\mathrm{ms}$ in the anterior medial temporal lobe (AMTL). Similar to the scalp-recorded N400, the AMTL-N400 is associated with semantic integration processes when visually presented words do not fit the preceding sentential context (McCarthy et al., 1995) or when words are possible, but semantically unexpected, in the context in which they appear (Kutas and Hillyard, 1984). The anterior part of the MTL is composed of the perirhinal and the entorhinal cortex, which are part of the parahippocampal region (Witter et al., 1989; Amaral and Insausti, 1990; Nobre et al., 1995). Anatomically, the perirhinal and parahippocampal cortices provide most of the neocortical input to the entorhinal cortex, which in turn provides the predominant cortical input to the hippocampus via the perforant path. There are also strong connections from the hippocampus back to the entorhinal cortex, to the perirhinal and parahippocampal cortices, and to neocortex (Witter et al., 1989; Amaral and Insausti, 1990; Lavenex and Amaral, 2000).

Syntax is composed of rules, including operation and constraints, which underlie the productive sequential and hierarchical combination of lexical forms and abstract representations into complex structures, including words, phrases, and sentences (Ullman, 2001). The involvement of the MTL in syntactic processing has not been discussed so far. A most recent imaging study, however, reported activation of the hippocampus during early stages of syntax learning, but not during proficient syntax processing (Opitz and Friederici, 2003). Thus, it is unclear to what extent the hippocampus is involved when syntax is processed in the adult proficient listener.

In ERP research, syntactic processing problems are associated with two components in the ERP recorded with scalp electrodes. Outright syntactic violations have often been reported to elicit a left anterior negativity (Friederici et al., 1993; Münte et al., 1993). 
Recent data from MEG recordings suggest that neural generators of this syntactic negativity reside in the left anterior temporal cortex and the inferior frontal gyrus (Friederici et al., 2000). Moreover, a late component, the so-called P600, is elicited by outright syntactic violations such as phrase structure errors (Neville et al., 1991; Osterhout and Holcomb, 1992, 1993; Friederici et al., 1996) and by violations of structural preferences as for example in garden-path sentences. This component is assumed to reflect late syntactic integration difficulties that require controlled processes of syntactic reanalysis and repair arising from syntactic anomaly or syntactic complexity (Osterhout and Holcomb, 1992; Friederici, 2002). Although a recent study demonstrated that the P600 elicited by syntactic violations has a more posterior scalp distribution than the P600 elicited by violations of structural ambiguities (Friederici et al., 2002), our current knowledge regarding the neuronal generators of the P600 is limited.

Based on the above-mentioned patient and intra- and extracranial ERP data, we examined whether there is a dissociation between the hippocampus proper and the rhinal cortex concerning the sensitivity to semantic and syntactic violations in natural speech.

Specifically, the hippocampus may be sensitive to particular syntactic aspects of speech, such as controlled reanalysis processes as could be inferred from H.M.'s language-processing deficits, whereas the rhinal cortex appears to be sensitive to semantic processes. To test this hypothesis, we recorded ERPs from intracranial electrodes, placed directly in the MTL of patients with pharmacoresistant temporal lobe epilepsy undergoing invasive presurgical evaluation, while the patients listened to sentences that either were correct or contained a syntactic or semantic violation. In contrast to scalp-recorded ERPs whose generators are difficult to localize, ERP recordings from intracranial electrodes have the appropriate spatial resolution to allow inferences about the cognitive processes mediated by the underlying neuronal structures (Bullock et al., 1995). Concerning the substructures of the MTL, such a separation would be impossible with surface electroencephalographic (EEG) recordings. The sensitivity of intracranial electrodes is maximal for field potentials generated within the adjacent brain tissue and, in general, decays with the inverse square of the distance (Morris and Luders, 1985). Previous studies using intracranial ERP data indicate that there is no detectable correlation between data recorded within the hippocampus and the rhinal cortex, even with electrode distances of $<1 \mathrm{~cm}$ (McCarthy et al., 1995; Fernández et al., 1999). Moreover, hippocampal activity is shielded toward the outside by the radial cylindrical arrangement of hippocampal pyramidal layers (Klee and Rall, 1977) and thereby impossible to record with surface EEG methods.

It has been shown that in the case of declarative memory formation, a direct interaction between the rhinal cortex and the hippocampus may be accomplished by phase synchronization of gamma activity between the two structures (Fell et al., 2001). Successful as opposed to unsuccessful memory formation was accompanied by an initial elevation of rhinal-hippocampal gamma synchronization followed by a later desynchronization, suggesting that effective declarative memory formation is accompanied by a direct and temporarily limited cooperation between these MTL substructures. The increased gamma phase coupling may reflect a change in the functional connectivity between rhinal and hippocampal regions that is important for initiating encoding processes, for instance, by facilitating the transmission of information between these regions. Subsequent desynchronization may mark termination of interactions following information transfer.

Phase synchronization of gamma oscillations (electrical brain activity) at $\sim 40 \mathrm{~Hz}$ is a general mechanism underlying transient functional coupling of neural assemblies (Varela et al., 2000; Engel and Singer, 2001). This mechanism provides an explanation for the flexibility and specificity of the functional associations between brain modules by enabling effective coupling and decoupling within as well as between different subsystems of the brain.

The results of several studies (Hirai et al., 1999; Fell et al., 2001) support the view that high-frequency oscillations of $\sim 40$ $\mathrm{Hz}$ have a prominent involvement in MTL, and especially hippocampal information processing. Furthermore, it has been proposed that the frequency range in which the functional coupling between two brain regions takes place is related to the distance between the regions, with proximate regions being more likely to be coupled by high EEG frequencies, whereas long-distance interareal interactions are reflected in lower frequencies (Sarnthein et. al, 1998). Because of the proximity of the MTL substructures, our analysis of functional coupling focused on high-frequency gamma oscillations. We hypothesized that syntactic errors may lead to an initial increase in synchronization to subserve information transfer from rhinal cortex to hippocampus, reflecting the selective involvement of the hippocampus in syntactic revision. The transfer could be conceived as the subordinate rhinal cortex making available semantic information in the services of comprehension and reanalysis. A similar view was put forward by Fernández and colleagues (2002) concerning the support operation of the parahippocampal region in declarative memory formation. To the extent to which semantic integration can be accomplished by the rhinal cortex on its own, we do not expect an increase of phase synchronization as no information needs to be transferred from rhinal cortex to hippocampus.

\section{MATERIALS AND METHODS}

\section{Subjects}

We investigated 10 patients. Because of extensive artifacts, one subject had to be excluded. All remaining nine patients (five women, four men; mean age: 36.1 years; range: $22-50$ years) had pharmacoresistant temporal lobe epilepsies (mean duration of illness: 22.5 years; range: $8-33$ years). All were native German speakers and right-handed. During presurgical evaluation, spontaneous seizures were recorded invasively using bilateral MTL 
depth electrodes in all patients and additional temporolateral strip electrodes in four patients. In five patients, seizures originated exclusively from the right MTL; in four patients, exclusively from the left MTL. The EEG study was approved by the local medial ethics committee. Each patient gave written informed consent.

\section{Experiment}

The experiment included a total of 192 sentences, half of which contained a violation. There were four types of experimental sentences: correct sentences (e.g., Die Tür wurde geschlossen, loosely translated: "The door was being closed"); semantically incorrect sentences in which the participle could not be integrated into the preceding sentence fragment as the context violated the selectional restrictions of the verb (Der Ozean wurde geschlossen, i.e., "The ocean was being closed"); syntactically incorrect sentences that contained a phrase structure error (Das Geschäft wurde am geschlossen, i.e., "The shop was being on closed"); and correct filler sentences containing a full prepositional phrase (Der Laden wurde am Samstag geschlossen, i.e., "The store was being on Saturday closed"). Syntactically incorrect sentences contained a phrase structure error. In these sentences, a preposition appeared after the auxiliary and was directly followed by a past participle. Because the preposition indicates the beginning of a prepositional phrase necessarily consisting of a preposition and a noun phrase, this sequence of words creates a clear word category violation. None of the violation sentences could be reinterpreted as coherent German sentences. The filler sentences were not included in the EEG analyses.

The study was conducted in a special unit for simultaneous video and EEG monitoring, with the patient sitting upright in an adjustable bed. All sentences were presented auditory via headphones (spoken by a trained female speaker). To ensure analogous prosody on the target (past participle) in all sentences, the syntactically incorrect sentences were recorded with a full prepositional phrase, including a noun after the preposition. This noun was later spliced out of the digitized speech signal, using a speech-editing tool. To ensure that this splicing would not be affected by coarticulation phenomena, we used nouns for which the phonological transitions from preposition offset to noun onset and from noun offset to participle onset were identical.

The mean sentence duration was $1,700 \mathrm{~ms}$. At $3 \mathrm{~s}$ after the final verb, the patient's task was to indicate, via mouse button press, whether a sentence contained a violation. The 3,000-ms interval was chosen to minimize the risk of movement artifacts in the ERP epoch used for analysis. The onset of the critical past participle verb was determined by visual and auditory inspection of the speech signal to allow for a precise time-locking of the ERPs of each sentence.

\section{EEG Recording}

Bilateral depth electrodes with a cylindrical surface area of $10 \mathrm{~mm}^{2}$ were implanted stereotactically along the longitudinal axis of the hippocampus from an occipital approach with the amygdala as the target for the most anterior electrode during presurgical evaluation. Implantation of intracranial electrodes was necessary because the zone of seizure onset could not be determined unequivocally by noninvasive investigations (Van Roost et al., 1998).

Each catheter-like, 1-mm-thick Silastic electrode contained 10 cylindrical contacts of a nickel-chromium alloy $(2.5 \mathrm{~mm})$ every $4 \mathrm{~mm}$. To determine the anatomical positions of electrode contacts, magnetic resonance imaging (MRI) scans were acquired in sagittal and adjusted coronal planes, perpendicular to the longitudinal axis of the hippocampus. Electrode contacts were mapped by transferring their positions from MRI to standardized anatomical drawings to facilitate identification of the electrode placement in each individual. Depth EEGs were referenced to linked mastoids, bandpass-filtered $(0.03-85 \mathrm{~Hz}$, $12 \mathrm{~dB}$ /octave), and recorded with a sampling rate of $173 \mathrm{~Hz}$ (12-bit analog-digital conversion). Details of implantation electrode localization and recording procedures were reported by Elger et al. (1997).

\section{Data Analysis \\ ERP data}

Since ERP potentials from the MTL are reduced near epileptogenic foci (Grunwald et al., 1998b), ERPs were computed only for the hemisphere in which no seizures were recorded. Epoch length was $800 \mathrm{~ms}$ starting with the onset of the final word of the sentence. By analyzing only EEG recordings from the MTL contralateral to the zone of seizure origin, we aimed at reducing poorly controllable effects induced by the epileptic process (Paller et al., 1992). Moreover, none of the MTLs investigated in this study showed any pathology, such as hippocampal atrophy, on clinical MRI scans performed during the presurgical workup. Since any prestimulus baseline would cover different word types, the first $50 \mathrm{~ms}$ after the onset of the critical word was used as a baseline to minimize the influence of the preceding word.

In order to examine whether the ERPs elicited by the violation conditions varied as a function of lesion side, in a first step, the ERP waveforms elicited in the left $(n=5)$ and right $(n=4)$ MTL lobes were directly contrasted. As the main focus of the present study was on syntactic and semantic violations, this analysis of hemispheric differences was performed for the two violation conditions. As no significant effects of hemisphere were obtained in the early and late time windows (Mann-Whitney test, $P$-values $=0.90$ and 0.19 , respectively), all the following analyses were conducted for the entire group of patients.

To test our hypothesis of a differential involvement of the rhinal cortex and the hippocampus proper in semantic and syntactic processing, analysis of variance (ANOVA) was performed with the factors ELECTRODE (2 levels: AMTL vs. MTL), CONDITION (3 levels: semantic incorrect vs. syntactically incorrect vs. correct), and TIME WINDOW (2 levels: early [200-500 ms] vs. late [500-800 ms]). As critical electrodes, two 
contacts were included in the ANOVA. For the rhinal cortex, the contact with the largest negative deflection at 200-500 ms after the onset of the semantic violation was selected; for the hippocampus, the contact with the largest negative deflection at 500$800 \mathrm{~ms}$ after the onset of the syntactic violation was selected. Previous studies showed that the AMTL-N400 has its largest amplitude in the early latency range (Grunwald et al., 1998a), whereas the later time window covers the temporal variability of the scalp-recorded P600 elicited by syntactic violations (Osterhout and Holcomb, 1992).

\section{EEG data: phase synchronization}

To obtain an optimal time and frequency resolution within the gamma band, a wavelet technique was applied to the EEG data. The same electrode positions as in the ERP analysis were used. The EEG was wavelet-filtered in the gamma frequency range from 32 to $48 \mathrm{~Hz}$ (2-Hz steps), implementing Morlet wavelets of 7 cycles length. As we were interested in induced gamma activity, analysis was based on single-trial evaluations to prevent the jittering induced response from being cancelled out in the time-domain average (Tallon-Baudry and Bertrand, 1999). Phase synchronization values between electrode contacts within the rhinal cortex and the hippocampus were calculated from the individual wavelet-transformed EEG segments (see Fell et al., 2001, for details related to the analysis of phase synchronization).

Finally, phase synchronization values were averaged for each experimental condition in consecutive time windows of $50-\mathrm{ms}$ duration from 50 to $750 \mathrm{~ms}$ (14 time windows in total). The first $(0-50 \mathrm{~ms})$ and the last time window $(750-800 \mathrm{~ms})$ were excluded because of edge effects.

Synchronization values were then normalized by dividing the condition mean value of each patient and time window by the patients' global mean value across all conditions, frequencies, and time windows. One patient had to be excluded from this latter analysis because most trials were contaminated with highfrequency noise.

\section{RESULTS}

\section{Behavioral Data}

All patients were highly accurate in classifying the sentences. The proportion of correct responses was $0.92(\mathrm{SEM}=0.03)$ for correct sentences, 0.94 (SEM $=0.03$ ) for semantically incorrect sentences and $0.93(\mathrm{SEM}=0.03)$ for syntactically incorrect sentences.

\section{ERP data}

As illustrated in Figure 1, both semantic violations and correct sentences elicited a large negative component at recording sites in the rhinal cortex with a maximum amplitude at 200500 ms. No such component was obtained for syntactic violations.
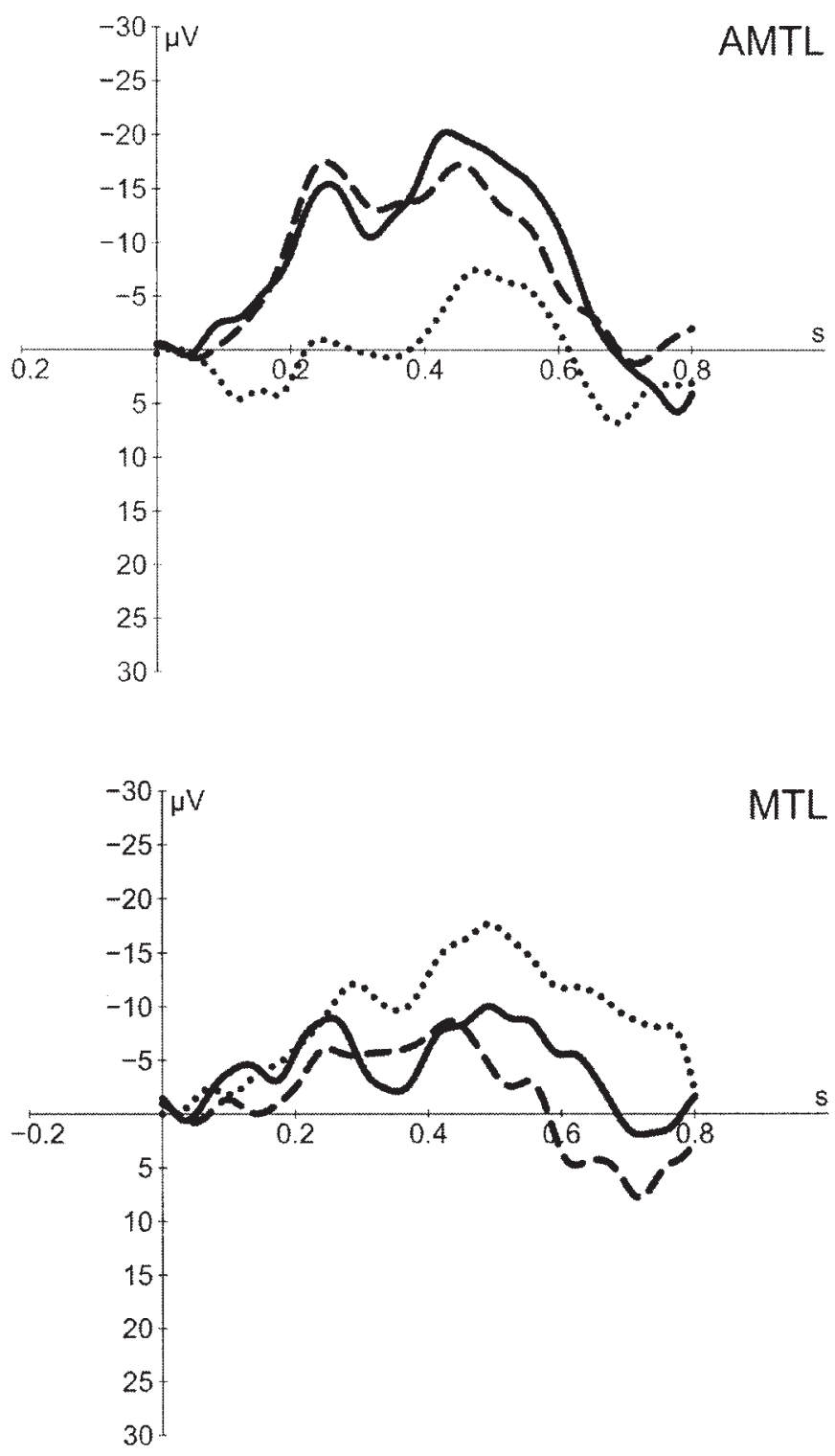

$$
\begin{aligned}
& \text { Legend: } \\
& \begin{array}{l}
\text { - Syn. Incor. }(n=9) \\
\text { - Sem. Cor. }(n=9) \\
- \text { Sem. Incor. }(n=9)
\end{array}
\end{aligned}
$$

FIGURE 1. Grand average event-related potentials (ERPs). Data from a contact in the anterior parahippocampal gyrus as ascertained by magnetic resonance imaging (MRI) with the maximal AMTL-N400 and from a contact in the hippocampus as ascertained by MRI with the maximal MTL-P600. Vertical axis denotes the onset of the critical sentence final word.

At the hippocampal recording sites, syntactic violations elicited a large negative deflection with a maximum amplitude at 500-800 ms. This component was absent for the semantically violated and the correct sentences.

Three-way ANOVA revealed an interaction between CONDITION and TIME WINDOW, $\mathrm{F}_{(2,16)}=3.62, P<0.05$, and an interaction between CONDITION and ELECTRODE, $\mathrm{F}_{(2,16)}=11.55, P<0.0009$. The three-way interaction did not reach significance, $\mathrm{F}_{(2,16)}=1.02, P>0.38$. 
correct sentences

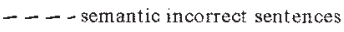

......syntactic incorrect sentences
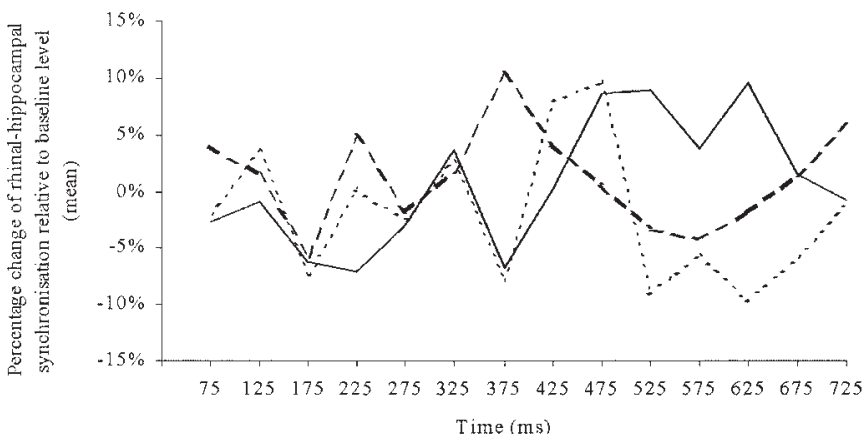

Time (ms)

FIGURE 2. Changes of phase synchronization between rhinal cortex and hippocampus (\%) relative to baseline for syntactically incorrect, semantically incorrect, and correct sentences. Synchronization values were averaged across all analyzed gamma band frequencies $(32-48 \mathrm{~Hz})$.

To resolve these interactions, follow-up analyses were performed separately for each of the electrodes. At the AMTL electrode, there was a significant difference in the early time window between the syntactic violation sentences and both correct and semantic violation sentences, $\mathrm{F}_{(1,8)}=5.21, P<0.05$ and $\mathrm{F}_{(1,8)}=10.07, P<0.02$. No corresponding effect was obtained in the late time window, $P$-values of $>0.05$.

Conversely, for the hippocampal electrode, no significant differences between the conditions were obtained for the early time window, $P$-values of $>0.05$, whereas a significant difference between the syntactic and the semantic violation was obtained in the late time window for the semantic-syntactic comparison, $\mathrm{F}_{(1,8)}=7.68, P<0.03$. The difference between the correct and the syntactically violated sentences was marginally significant, $\mathrm{F}_{(1,8)}=3.73, P<0.09$, whereas the correct and the semantically violated sentences did not differ from each other at 500-800 ms, $P>0.05$.

To summarize briefly, the main result of the present study is that sentences carrying a syntactic violation elicited a large negative response at hippocampal recording sites with its maximum at $\sim 500 \mathrm{~ms}$, but no significant response in the rhinal cortex. In contrast, sentences with a semantic violation and correct sentences elicited an earlier peaking AMTL-N400 within the rhinal cortex, but no response at hippocampal recording sites.

\section{Phase Synchronization}

As is apparent from Figure 2, the phase synchronization values for the two violation conditions were enhanced in two time windows: first, at 350-400 ms, where semantically incorrect sentences showed an increase of phase synchronization of $\sim 10 \%$ relative to baseline, and second, $100 \mathrm{~ms}$ later at 450 $500 \mathrm{~ms}$, where now syntactically incorrect sentences exhibited an enhancement of phase synchronization of $\sim 10 \%$ relative to baseline. In contrast, correct sentences were related with a long-lasting increase of phase synchronization relative to base- line starting at $\sim 500 \mathrm{~ms}$ that reached its maximum at 600 $650 \mathrm{~ms}$.

These observations were confirmed by statistical analyses. ANOVA contrasting the two violation conditions in the 350400-ms time interval revealed a marginally significant difference between the conditions, $\mathrm{F}_{(1,7)}=4.95, P<0.06$.

Conversely, phase synchronization at 450-500 ms was higher for syntactic violations than for semantic violations $\mathrm{F}_{(1,7)}=$ 7.97, $P<0.02$. Finally, the correct sentences significantly differed from the syntactically violated sentences for the time window between 600 and $650 \mathrm{~ms}, \mathrm{~F}_{(1,7)}=10.31, P<0.02$.

\section{DISCUSSION}

We found a dissociation between rhinal cortex and hippocampus proper concerning the two types of violation of spoken language. This raises the issue of the detailed processing function of both structures in language comprehension. Second, it remains to be evaluated how these processing functions are related to those underlying declarative memory.

\section{Semantic Processes}

Converging evidence from single-cell recordings, lesion studies, depth ERP, and imaging studies (e.g., Grunwald et al., 2003; for review, see Brown and Aggleton, 2001) suggest that specific processing functions can be attributed to the different substructures of the hippocampal formation. With respect to the rhinal cortex, it is argued that it is operating in a fast and automatic manner and its representational format is restricted to single items (Eichenbaum et al, 1999) or overlearned combinations of items (Naya et al., 2001, 2003; O'Reilly and Norman, 2002). It receives input from multiple sensory areas and likely plays a role in object perception and identification by binding the various attributes of an object into a reified representation (Murray and Richmond, 2001). These less complex associations suffice for the representations of factual (i.e., semantic) information (Mishkin et al., 1998). They develop by processing similar inputs in different episodic contexts over a specific period. One could speak of intersections of similar episodic events. Neurons in the rhinal cortex signal the novelty, recency, or familiarity of individual items. This ability permits the rhinal cortex to subserve the familiarity and recency discrimination components of recognition memory (Brown and Aggleton, 2001) and may also play an important role for semantic memory (Varga-Kahdem et al., 1997; Mishkin et al., 1998; Fernández et al., 2002).

The N400 appears to reflect the use of semantic memory during language comprehension. A word embedded in a sentence takes its meaning from the knowledge stored in the brain through a lifetime of experiences. This knowledge is organized in semantic memory along the dimensions of similarity and association, and it is this structure of semantic memory that influences on-line language processing. The N400 amplitude is sensitive to the ease of accessing information from semantic 
long-term memory. For example, N400 amplitudes to words presented out of context vary as a function of the frequency of usage (Kutas and Federmeier, 2000). The present findings confirm and extend previous studies reporting AMTL-N400s for verbal stimuli in the visual domain (Elger et al., 1997; Grunwald et al., 1998b), pointing to the supramodal nature of the semantic integration processes.

In addition, the present results provide compelling evidence that the detection of a syntactic error eliminates the AMTLN400, compatible with findings from scalp-recorded ERP research (Friederici et al., 1999a). In contrast to a variety of studies with scalp ERPs using the same stimulus materials with which an attenuated N400 for correct sentence endings compared with semantically incorrect endings was observed (Friederici et al., 1993; Hahne and Friederici, 2001), our experiment revealed an AMTL-N400 for both, for sentences containing a semantic violation and for correct sentences. Before drawing any firm conclusions from this discrepancy between the scalp-recorded N400 and the AMTL N400, it has to be acknowledged that the large majority of N400 studies on semantic integration investigated normal adults without any history of neurological diseases, whereas in the present study we examined patients with a long history of temporal lobe epilepsy that may have altered the brain systems mediating semantic processing.

A hint toward the understanding of this functional dissociation between the AMTL N400 and the scalp-recorded N400 can be derived when comparing sentence studies with studies probing semantic integration with single words as contextual cues. These studies reveal attenuated scalp N400 components (Holcomb and Neville, 1990) as well as AMTL N400 (Nobre and McCarthy, 1995) to semantically primed words.

Given this, it is conceivable that the observed dissociation between the N400 and the AMTL N400 is restricted to semantic integration in sentence contexts. While word-priming studies evaluated the relationship between two nouns, sentence studies such as the present one focused on the relationship between a noun and a verb. Contextual information provided by sentences thus may activate widely distributed, spatiotemporal dynamic neocortical processing systems (Halgren et al., 2002), leading to facilitated integration of correct sentence terminal words as reflected by the attenuated N400. Those involved cortical areas might have different functional properties and are therefore specialized for different aspects of the process of semantic contextual integration (Halgren, 1990). Conversely, the rhinal cortex may not be sensitive to sentential context relevant to the relationship between verbs and their noun arguments, leading to large AMTL N400 irrespective of the semantic correctness of the sentence terminal word.

\section{Syntactic Processes}

The hippocampus proper, as compared with the rhinal cortex, is hypothesized to work more slowly (Fernández et al., 1999), and in an associational manner (Eichenbaum, 2000). This means that its representational format is not restricted to single items but, unlike the rhinal cortex, it is capable of representing many items and many inputs from different cortical regions, and most importantly their relationships to each other (McClelland et al., 1995). Thus, the hippocampus is able to bind contextually the specific features of an event like time, place, and content to an episode (Squire and Knowlton, 2000; Eichenbaum and Cohen, 2001) or an utterance and to subserve memory encoding, consolidation, and integration of given information into stored knowledge. The processing of syntactic violations as indexed by the negative component in the hippocampus can be conceived of as the late consequence of an unsuccessful template matching process between a small set of fixed structures stored in syntactic memory and a current, just perceived input. For example, the syntactic structure of a prepositional phrase is fixed, as it always contains a preposition followed by a noun phrase. Once the element following the preposition is not part of a noun phrase (as present in the syntactic violation condition), the incoming information does not match the phrase structure template. This early matching procedure is automatic, may be more reliant on neocortical brain areas, and is reflected by an early left anterior negativity (ELAN) in the scalp-recorded ERP or MEG measures (Friederici et al., 1999b, 2000). After detection of the syntactic violation, controlled reanalysis and repair processes come into play, which may depend on declarative memory (Ullman, 2001). There are no automatic procedural processing steps determining the handling of the unfamiliar sentence material. The processes involved in syntax comprehension seem to rely on brain structures separate from the areas in which syntax representations themselves or rules reside (Patel, 2003). Indeed, a recent functional magnetic resonance imaging (fMRI) study (Friederici et al., 2003) using the same sentence material as the present experiment identified the frontal operculum in the inferior frontal cortex, and specifically the left anterior and posterior portion of the superior temporal gyrus (STG) to support syntactic processes, whereas semantic processes were supported by the middle and posterior portion of the STG. Given that the early syntax-related template-matching processes were associated with two dipoles, located within the inferior frontal cortex and the anterior temporal lobe, the posterior temporal region represents a good candidate for the late integration process reflected in the P600. This notion is supported by a recent ERP study with patients suffering from lesions in the left posterior temporal lobe. These patients do not show a P600, although an early left anterior negativity (Friederici and Kotz, 2003). Thus, the presently observed dissociation together with those reported from lesion patients can be understood as the distinction between long-term structural knowledge in a given domain and operations conducted on that knowledge (for a related model of the P600, see Hagoort, 2003).

Syntax may be defined as a set of principles governing the combination of discrete structural elements (e.g., words) into sequences (Jackendoff, 2002). Thus, the rules of grammar have per se a relational character, but they are well consolidated during development and are applied in an automatic manner. According to the declarative/procedural model recently pro- 
posed by Ullman (2001), it is the procedural memory system, which is composed of a network of specific frontal, basal-ganglia, parietal, and cerebellar structures, and which supports the learning and execution of motor and cognitive skills, especially those involving sequences, that represents the rules of grammar. So, a new and unfamiliar linguistic input, as in the case of a phrase structure violation, requires a new relational moment. In that sense, each sentence may be seen as having some kind of episodic value. In this framework, it could be argued that sentences with a syntactic violation cannot be represented with the acquired rules, with the consequence that known words have to be bound in a novel, grammatically unknown manner. They may thus give rise to increased binding demands and by this activate the hippocampus. In other words, they necessitate a shift back to the declarative memory system what in turn is observable in a P600, which is associated with controlled processing. (Ullman, 2001).

The assumed relationship between the hippocampus and the late syntactic integration processes reflected by the P600 predicts a syntactic processing deficit in patients with hippocampal lesions, which could recently be confirmed in a further study (P. Meyer, unpublished data). We tested one of the patients in the present study after epilepsy surgical resection of his left hippocampus and amygdala with the same sentence material. In scalp-recorded ERPs, we found a selective reduction of the P600 component over the left side of the left hemisphere for the syntactic violation condition, relative to a control group, matched for age and education, pointing to the hippocampus as a member of the P600 generating network. Notably, the N400 did not differ between the patient and his controls. Another relevant structure of the P600 generating network are the basal ganglia. ERP studies with patients suffering from lesions in the left basal ganglia showed a reduced P600 (Frisch et al., 2003). Thus, the hippocampus together with the posterior STG and the basal ganglia appear to constitute major structures supporting the processes reflected in the P600.

It is conceivable that the hippocampus is needed to perform a context-dependent relation rearrangement among the single items (words in this case) to allow the representation and, hence, at least in the case of syntactic infrequent structures like gardenpath sentences, the understanding of a nonpreferred syntactical structure. Thus, the hippocampal representational format is not only involved in episodic memory, but rather influences language processing as well. This suggests that analogous computations in the hippocampus may underlie a range of cognitive domains. The language-related function of the hippocampus thus seems to be highly related to its nonlanguage functions. Hippocampal lesions may, in turn, lead to an impairment that was labeled "binding syndrome" (MacKay et al., 1998), which can explain both episodic memory deficits and language-related deficits as for example found in patient H.M.

Our predictions concerning phase synchronization between perirhinal and hippocampal structures were not completely confirmed by the observed data. An increase of gamma phase synchronization at $\sim 500 \mathrm{~ms}$ could be detected for the syntax violations. However, there also was a phase synchronization increase for the semantically violated sentences at $\sim 400 \mathrm{~ms}$ that we did not expect to find. Interestingly, in showing maximal increase in phase synchronization at 350-400 ms and 450-500 ms, phasic enhancement of phase synchronization in both conditions was temporarily correlated with the measured ERPcomponents. Consistent with prior studies on declarative memory encoding (Fernández et al., 1999; Fell et al., 2001), the present phase synchronizations preceded the respective ERP components. The correct sentences, however, showed a longlasting increase in phase coupling relative to baseline starting at $\sim 500 \mathrm{~ms}$. If the processing and understanding of a correct sentence are associated with such a late synchronization pattern between the rhinal cortex and the hippocampus, it may be argued that, in the case of a violation, the normal increase in phase coupling during a late time window stops when semantic or syntactic integration problems have be dealt with. This late time window can be thought to cover the process of integrating semantic and syntactic information to achieve sentence comprehension (Gunter et al., 2000). The clear temporal structure of the increase of synchronization, i.e, $350-400 \mathrm{~ms}$ for the semantically incorrect condition, 450-500 $\mathrm{ms}$ for the syntactically incorrect condition, and beyond $500 \mathrm{~ms}$ for the correct condition, provides initially indication for sequential nature of different aspects of sentence processing. Even though the exact relationship between phase synchronization and ERP components remains to be specified, the present data suggest that regular sentence processing is associated with functional coupling of both structures through gamma synchronization. Both semantic and syntactic violations, however, seem to interrupt this rhinal-hippocampal interplay, perhaps in order to prevent unnecessary further processing and encoding of an erroneous sentence into declarative memory.

Taken together, our results provide compelling evidence for a differential sensitivity of MTL substructures for different integration processes in natural language comprehension. Syntactic violations elicited an MTL-P600 in the hippocampus proper, but no AMTL-N400 in the rhinal cortex. Conversely, this latter rhinal negative potential was present in semantically incorrect sentences. An analysis of phase synchronization within the gamma band between rhinal and hippocampal recording sites shows that both ERP components are preceded by increases of phase synchronization between the MTL structures. Moreover a long-lasting increase of phase synchronization during a late time window is found in the processing of correct sentences that is presumably suspended in case of semantic and syntactic violations. These data indicate that the hippocampus proper should be considered as part of the neural network subserving processes of late syntactic integration as reflected by the P600.

\section{REFERENCES}

Amaral DG, Insausti R. 1990. Hippocampal formation. In: Paxinos, G, editor. The human nervous system. San Diego, CA: Academic Press. p 711-755. 
Brown MW, Aggleton JP. 2001. Recognition memory: what are the roles of the perirhinal cortex and hippocampus? Nat Rev Neurosci 2:51-61.

Bullock TH, McClune MC, Achimowicz JZ, Iragui-Madoz VJ, Duckrow RB, Spencer SS. 1995. EEG coherence has structure in the millimetre domain: subdural and hippocampal recordings from epileptic patients. EEG Clin Neurophysiol 95:161-177.

Eichenbaum H. 2000. Cortical-hippocampal networks for declarative memory. Nat Rev Neurosci 1:41-50.

Eichenbaum H, Cohen NJ. 2001. From conditioning to conscious recollection: memory systems of the brain. New York: Oxford University Press.

Eichenbaum H, Dudchenko P, Wood E, Shapiro M, Tanila H. 1999. The hippocampus, memory and place cells: is it spatial memory or a memory space? Neuron 23:209-226.

Elger CE, Grunwald T, Lehnertz K, Kutas M, Helmstaedter C, Brockhaus A, Van Roost D, Heinze H. 1997. Human temporal lobe potentials in verbal learning and memory processes. Neuropsychologia 35:657-668.

Engel AK, Singer W. 2001. Temporal binding and the neural correlates of sensory awareness. Trends Cogn Sci 5:16-25.

Fell J, Klaver P, Lehnertz K, Grunwald T, Schaller C, Elger CE, Fernández G. 2001. Human memory formation is accompanied by rhinalhippocampal coupling and decoupling. Nat Neurosci 4:1259-1264.

Fernández G, Effern A, Grunwald T, Pezer N, Lehnertz K, Dümpelmann M, Van Roost D, Elger CE. 1999. Real-time tracking of memory formation in the human rhinal cortex and hippocampus. Science 285:1582-1585.

Fernández G, Klaver P, Fell J, Grunwald T, Elger CE. 2002. Human declarative memory formation: segregating rhinal and hippocampal contributions. Hippocampus 12:514-519.

Friederici AD. 2002. Towards a neural basis of auditory sentence processing. Trends Cogn Sci 6:78-84.

Friederici AD, Kotz SA. 2003. The brain basis of syntactic processes: functional imaging and lesion studies. NeuroImage 20(Spec Issue):8-17.

Friederici AD, Pfeifer E, Hahne A. 1993. Event-related brain potentials during natural speech processing: effects of semantic, morphological and syntactic violations. Cogn Brain Res 1:183-192.

Friederici AD, Hahne A, Mecklinger A. 1996. Temporal structure of syntactic parsing: early and late event-related brain potential effects elicited by syntactic anomalies. J Exp Psychol Learn 22:1219-1248.

Friederici AD, Steinhauer K, Frisch S. 1999a. Lexical integration: sequential effects of syntactic and semantic information. Mem Cognit 27:438-453.

Friederici AD, von Cramon DY, Kotz SA. 1999b. Language related brain potentials in patients with cortical and subcortical left hemisphere lesions. Brain 122:1033-1047.

Friederici AD, Wang Y, Herrmann CS, Maess B, Oertel U. 2000. Localization of early syntactic processes in frontal and temporal cortical areas: a magnetoencephalographic study. Hum Brain Mapp 11:1-11.

Friederici AD, Hahne A, Saddy D. 2002. Distinct neurophysiological patterns reflecting aspects of syntactic complexity and syntactic repair. J Psycholinguist Res 31:45-63.

Friederici AD, Rüschemeyer SA, Hahne A, Fiebach CJ. 2003. The role of the left inferior frontal and superior temporal cortex in sentence comprehension: localizing syntactic and semantic processes. Cereb Cortex 13:170-177.

Frisch S, Kotz SA, von Cramon DY, Friederici AD. 2003. Why the P600 is not just a P300: the role of the basal ganglia. Clin Neurophysiol 114:336-340.

Grunwald T, Lehnertz K, Helmstaedter C, Kutas M, Pezer N, Kurthen M, Van Roost D, Elger CE. 1998a. Limbic ERPs predict verbal memory after left-sided hippocampectomy. NeuroReport 9:3375-3378.

Grunwald T, Lehnertz K, Heinze HJ, Helmstaedter C, Elger CE. 1998b. Verbal novelty detection within the human hippocampus proper. Proc Natl Acad Sci USA 95:3193-3197.
Grunwald T, Pezer N, Münte T, Kurthen M, Lehnertz K, Fernández G, Kutas M, Elger CE. 2003. Dissecting out conscious and unconscious memory (sub)processes within the human medial temporal lobe. NeuroImage 20(S1):139-145.

Gunter TC, Friederici AD, Schriefers H. 2000. Syntactic gender and semantic expectancy: ERPs reveal early autonomy and late interaction. J Cogn Neurosci 12:556-568.

Hagoort P. 2003. How the brain solves the binding problem for language: a neurocomputational model of syntactic processing. NeuroImage 20(S1):S18-29.

Hahne A, Friederici AD. 2001. Differential task effects on semantic and syntactic processes as revealed by ERPs. Cogn Brain Res 13:339-356.

Halgren E. 1990. Insights from evoked potentials into the neuropsychological mechanisms of reading. In: Scheibel A, Weschsler A, eds. Neurobiology of cognition. New York: Guilford p 103-150.

Halgren E, Dhond RP, Christensen N, Van Petten C, Marinkovic K, Lewine JD, Dale AM. 2002. N400-like magnetoencephalography responses modulated by semantic context, word frequency, and lexical class in sentences. NeuroImage 17:1101-1116.

Hirai N, Uchida S, Maehara T, Okubo Y, Shimizu H. 1999. Enhanced gamma $(30-150 \mathrm{~Hz})$ frequency in the human medial temporal lobe. Neuroscience 90:1149-1155.

Holcomb P, Neville H. 1990. Auditory and visual semantic priming in lexical decision: a comparison using event-related brain potentials. Language Cogn Proc 4:281-312.

Jackendoff R. 2002. Foundations of language: brain, meaning, grammar, evolution. Oxford, UK: Oxford University Press.

Kensinger EA, Ullman MT, Corkin S. 2001. Bilateral medial temporal lobe damage does not affect lexical or grammatical processing: evidence from amnesic patient H.M. Hippocampus 11:347-360.

Klee M, Rall W. 1977. Computed potentials of cortically arranged populations of neurons. J Neurophysiol 40:647-666.

Kutas M, Dale A. 1997. Electrical and magnetic readings of mental function. In: Rugg MD, editor. Cognitive neuroscience. Hove East Sussex, UK: Psychology Press. p 197-242.

Kutas M, Hillyard SA. 1984. Brain potentials during reading reflect word expectancy and semantic association. Nature 307:161-163.

Kutas M, Federmeier AD. 2000. Electrophysiology reveals semantic memory use in language comprehension. Trends Cogn Sci 4:463-470.

Lavenex P, Amaral DG. 2000. Hippocampal-neocortical interaction: a hierarchy of associativity. Hippocampus 10:420-430.

MacKay D, Stewart R, Burke DM. 1998. H.M. revisited: relations between language comprehension, memory and the hippocampal system. J Cogn Neurosci 10: 3:377-394.

McCarthy G, Nobre AC, Bentin S, Spencer DD. 1995. Languagerelated field potentials in the anterior-medial temporal lobe. I. Intracranial distribution and neural generators. J Neurosci 15:1080-1089.

McClelland JL, McNaughton BL, O'Reilly RC. 1995. Why are there complementary learning systems in the hippocampus and neocortex: insights from successes and failures of connectionist models. Psychol Rev 102:419-457.

Mishkin M, Vargha-Khadem F, Gadian DG. 1998. Amnesia and the organization of the hippocampal system. Hippocampus 8:212-216.

Morris HH, Luders H. 1985. Electrodes. Electroencephalogr Clin Neurophysiol 37(Suppl):3-26.

Münte TF, Heinze HJ, Mangun GR. 1993. Dissociation of brain activity related to syntactic and semantic aspects of language. J Cogn Neurosci 5:335-344.

Murray EA, Richmond BJ. 2001. Role of perirhinal cortex in object perception, memory, and associations. Curr Opin Neurobiol 11:188-193.

Naya Y, Yoshida M, Miyashita Y. 2001. Backward spreading of memoryretrieval signal in the primate temporal cortex. Science 291:661-664.

Naya Y, Yoshida M, Miyashita Y. 2003. Forward processing of longterm associative memory in monkey inferotemporal cortex. J Neurosci 23:2861-2871. 
Neville HJ, Nicol J, Barss A, Forster KI, Garrett MF. 1991. Syntactically based sentence processing classes: evidence from event-related brain potentials. J Cogn Neurosci 3:151-165.

Nobre AC, McCarthy G. 1995. Language-related field potentials in the anterior-medial temporal lobe. II. Effects of the word type and semantic priming. J Neurosci 15:1090-1098.

Opitz B, Friederici AD. 2003. Interactions of the hippocampal system and the prefrontal cortex in learning language-like rules. NeuroImage 19:1730-1737.

O'Reilly RC, Norman KA. 2002. Hippocampal and neocortical contributions to memory: advances in the complementary learning systems framework. Trends Cogn Sci 6:505-510.

Osterhout L, Holcomb PJ. 1992. Event-related potentials and syntactic anomaly. J Mem Language 31:785-804.

Osterhout L, Holcomb PJ. 1993. Event-related potentials and syntactic anomaly: evidence of anomaly detection during the perception of continuous speech. Language Cogn Processes 8:413-437.

Paller KA, McCarthy G, Roessler E, Allison T, Wood CC. 1992. Potentials evoked in human and monkey medial temporal lobe during auditory and visual oddball paradigms. Electroencephalogr Clin Neurophysiol 84:269-279.

Patel AD. 2003. Language, music, syntax and the brain. Nat Neurosci 6:674-681.

Sarnthein J, Petsche H, Rappelsberger P, Shaw GL, von Stein A. 1998. Synchronization between prefrontal and posterior association cortex during human working memory. Proc Natl Acad Sci USA 95:7092-7096.
Squire LR, Knowlton BJ. 2000. The medial temporal lobe, the hippocampus, and the memory systems of the brain. In: Gazzaniga MS, editor. The new cognitive neurosciences. Cambridge, MA: MIT Press. p 765-780.

Tallon-Baudry C, Bertrand O. 1999. Oscillatory gamma activity in humans and its role in object representation. Trends Cogn Sci 3:151-162.

Ullman MT. 2001. The neural basis of lexicon and grammar in first and second language: the declarative/procedural model. Bilingualism Language Cogn 4:105-122.

Van Roost D, Solymosi L, Schramm J, Van Oosterwyck B, Elger CE. 1998. Depth electrode implantation in the length axis of the hippocampus for the presurgical evaluation of medial temporal lobe epilepsy: a computed tomography-based stereotactic insertion technique and its accuracy. Neurosurgery 43:819-826.

Varela FJ, Lachaux JP, Rodriguez E, Martinerie J. 2000. The brainweb: phase synchronization and large-scale integration. Nat Rev Neurosci 2:229-239.

Vargha-Khadem F, Gadian DG, Watkins KE, Connelly A, Van Paesschen W, Mishkin M. 1997. Differential effects of early hippocampal pathology on episodic and semantic memory. Science 277:376380 .

Witter MP, Groenewegen HJ, Lopes da Silva FH, Lohman AHM. 1989. Functional organization of the extrinsic and intrinsic circuitry of the parahippocampal region. Prog Neurobiol 33:161253. 